\title{
Proteome Analysis of Vernalization-Treated Arabidopsis thaliana by Matrix-Assisted Laser Desorption/Ionization Time-of-Flight Mass Spectrometry
}

\author{
Mi Ran Cho, Kyung Hyeon Lee, You-Bong Hyun, ${ }^{\dagger}$ Ilha Lee, ${ }^{\star}$ and Hie-Joon Kim* \\ Department of Chemistry, ${ }^{\dagger}$ Department of Biological Sciences, Seoul National Lniversity, Seoul 151-747, Korea \\ "E-mail: hifimlasulac.kr \\ Received October 26, 2006
}

\begin{abstract}
In order to gain insight into the molecular changes at the protein level in plants exposed to low temperature for a long period of time (vernalization) proteome analyses of vernalization-treated Arabidopsis thatiana have been carried out by two-dimensional gel electrophoresis followed by matrix-assisted laser desorption/ ionization time-of-flight mass spectrometry. Fourteen proteins including ATP binding/GTP binding/translation elongation factor and glycine-rich RNA-binding protein 7 (GRP7) showed differential expression in vernalization-treated Arabidopsis thaliana. GRP7 showed the most dramatic increase in expression suggesting its involvement in response to vernalization treatment.
\end{abstract}

Key Words : Arabidopsis thaliana. Vemalization. MALDI-TOF MS. GRP7

\section{Introduction}

Flowering time of plants is regulated by both endogenous chemical signals and envirommental cues. The two major environmental factors influencing flowering time are light and temperature. ${ }^{l}$ Many plants recognize a seasonal change for flowering through photoperiod. a length of day. Recent analysis showed that circadian rhythm of a gene called CONSTANS (CO) and coincidental light exposure at the peak time of $\mathrm{CO}$ is the molecular basis of photoperiodic flowering response in both monocots and dicots.'

Temperature is another important factor in determining the flowering time. It has been reported that flowering is accelerated in winter annuals of many plant species (such as wheat. barley cabbage and Arabidopsis) if exposed to low temperature for a long time. It is called vernalization. ${ }^{3}$ Vernalization is an evolutionarily advantageous mechanism preventing premature flowering during late fall and allowing flowering in the following spring in response to fasorable environments. ${ }^{3 .+}$ The effect of vernalization treatment depends on the growth status and genotype of the plant as well as the length of the treatment. ${ }^{3.4}$ The recent molecular genetic analyses of Arabidopsis winter annuals revealed the epigenetic regulatory mechanism of vernalization such that vernalization changes the chromatin structure of a strong floral repressor. FLOWERING LOCUSC (FLC) from active to silenced state. ${ }^{5}$ However. what causes such chromatin modification is not understood. Therefore. it is of critical importance to see proteomic changes induced by vernalization. It is hoped that the identification of proteins differentially expressed in vernalization-treated $A$. thaliona could provide a clue to understanding. at the molecular level. key steps in controlling the flowering time of plants in general. In this paper. we report identification. by 2-D electrophoresis and MALDI-TOF mass spectrometry (MS). of several proteins that seem to be associated with vernalization.

\section{Experimental Section}

Chemicals. Inmobilized $\mathrm{pH}$ gradient strips $(\mathrm{pH}$ 5-8. Ready Strip. $0.5 \times 3.3 \times 170 \mathrm{~mm}$ ). acrylamide. sodium dodecyl sulfate (SDS). anmonium persulfate. coomassie Brilliant Blue (CBB) R-250. 3-[(3-cholamidopropyl)dimethy lammoniol-1-propanesulfonate (CHAPS), protein assay solution. and ampholyte (Bio-Lyte. 3/10) were from Bio-Rad (Hercules. CA. USA). Urea thiourea. N.N'-methylenebisacrylamide. Triton X-100. dithiothreitol (DTT), tripsin, and iodoacetamide (IAA) were from Signa (St. Louis. MO. USA). Tris(hydroxymethyl)-aminomethane $\alpha$-cyano-4-hydroxy cinnamic acid (CHCA) were from Aldrich (Milwaukee. WI. USA).

Vernalization treatment. $A$. thaliona was grown under the long-day condition ( $16 \mathrm{~h}$ light period. $8 \mathrm{~h}$ dark period). After stratification for 3 days at $4{ }^{\circ} \mathrm{C}$. the control plant was grown for 11 days at $23^{\circ} \mathrm{C}$. Vernalization involved normal growth at $23^{\circ} \mathrm{C}$ for 9 days followed by 30 days at $4^{\circ} \mathrm{C}$.

Protein extraction and 2-D electrophoresis. The plant tissue was pulverized in liquid nitrogen using a mortar and pestle. Lysis buffer ( $6 \mathrm{M}$ urea. $2 \mathrm{M}$ thiourea. $50 \mathrm{mM}$ DTT. $2 \%$ CHAPS. $0.5 \%$ Triton X-100, $40 \mathrm{mM}$ Tris. $0.2 \%$ BioLyte) was added. and the mixture was vortex mixed for 30 min and centrifuged at $14.000 \mathrm{rpm}$ for $30 \mathrm{~min}$ at $4^{\circ} \mathrm{C}$. Protein was precipitated with $80 \%$ acetone for 1 h at $-20^{\circ} \mathrm{C}$. The protein pellet was washed with $80 \%$ acetone. dried. and dissolved in a minimum volume of the lysis buffer. The amount of protein in the supernatant was determined by the Bradford method.

Isoelectric focusing (IEF) followed by polyacrylamide gel electrophoresis was performed in a standard manner. Image Master $^{\mathrm{TM}}$ (Swiss Institute of Bioinformatics. Switzerland) was used to calculate the volume percent of the protein spots on the gel.

MALDI-TOF MS of tryptic peptides. The protein spot from the gel was sliced and digested overnight at $37^{\circ} \mathrm{C}$ with 
$10 \mu \mathrm{L}$ of a trypsin solution $(2 \mu \mathrm{g} / \mathrm{mL}$ in anumonium bicarbonate). For MS analysis. $1.5 \mu \mathrm{L}$ of the matrix solution ( $2 \mathrm{mg} \mathrm{CHCA}$ in $60 \% \mathrm{ACN}$ with $0.1 \% \mathrm{TFA}$ ) was mixed with an equal volume of the tryptic digest. and a $0.5 \mu \mathrm{L}$ aliquot was loaded on AnchorChip ${ }^{\mathrm{IM}}$ target plate. Auto Flex II MALDI-TOF MS/MS (Bniker Daltonics) equipped with a $337 \mathrm{~nm}$ nitrogen laser delayed extraction. and reflectron was used in the positive ion mode. MASCOT peptide mass fingerprint search program (http:/www.matrixscience.com/ cgi/search form.pl'?SEARCH=PMF) was used for protein identification. The search options were: NCBI database. Arabidopsis thaliana. maximum of 2 miss cleavages. 100 ppm mass error.

Transcriptional analysis. To characterize the transcriptional level of $A t G R P 7$, total RNA was extracted from 11 day's old seedlings using the RNeasy Plant Mini Kit (Qiagen. Valencia. CA). Through reverse transcription-polymerase chain reaction (RT-PCR) amplification, the expression level of each gene was analyzed. The sequences of primers used were AtGRP7F (TGCTTCGTTGGAGGTCTAGC)/AtGRP7R (GTAACCTCCTCCTTCACCAC) for $A t G R P 7$, and TUB-F (CTCAAGAGGTTCTCAGCAGTA)/TUB-R (TCACCTTCTTCATCCGCAGTT) for TUBULIN.

\section{Results and Discussion}

Results from 2-D electrophoretic separation of proteins extracted from control and vernalization-treated $A$. thationa are shown in Figure 1. Some distinct changes were evident by visual inspection. A total of 41 proteins were identified. Among them. 27 spots (indicated with arrows) were observed in both samples without major changes (Table 1). They include Rubisco activase. glutathione transferase nucleoside diphosphate kinase. and ribulose-bisphosphate carboxylase. In some cases the changes were certain but minor. In several cases. two spots separated horizontally were identified as the same protein $(1 / 2,3 / 4,9 / 10 / 11 / 15,13 / 14,23 / 24,25 / 26)$ and probably represent modification of the acidic or basic group resulting in the shift in isoelectric $\mathrm{pH}(\mathrm{pl})$ with a small change in the molecular weight. Most of the proteins not affected by vemalization correspond to the functional group of metabolism and indicate that vernalized plants carry out basic metabolic activity at the normal rate

A total of 14 protein spots (12 different proteins) showing increase or decrease in intensity by a factor of about two or more are circled. Again. proteins related by modification were observed $(3 / 4,5 / 6)$. These differentially expressed proteins are summarized in Table 2. Proteins showing decreased and increased expression are listed separately in the increasing order of differential expression. The percent volume of the protein spots in Table 2 are average of results from 16 separate gels (multiple harvests and extractions from control and vernalization-treated $A$. thaliona).

Eight proteins showing significant decrease in the expression level upon vemalization-treatment include glutathione transferase. translation elongation factor. transcription factor. putative major latex protein. glyceraldelyde 3-phosphate dehydrogenase. and carbonic anhydrase. Since the vemalization-treatment was essentially a cold-treatment. decrease in certain metabolic and regenerative proteins was expected. However. why these particular proteins are significantly lowered in cellular concentration in a reproducible manner is not understood and poses an interesting question in terms of their structure and function. The reproducibility in the decreased expression of putative major latex protein (spot 14 ) is shown in Figure 2 as an example. The observation was reproducible in duplicate extractions from two separate harvests of the plant.

We were more interested in finding proteins that are overexpressed in venalization-treated $A$. thaliana. Three pro- (a)

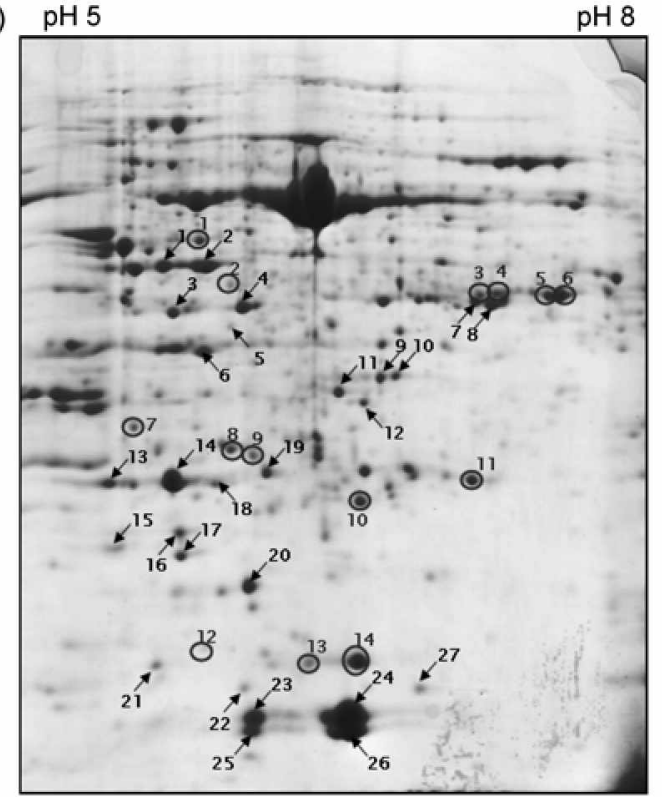

(b)

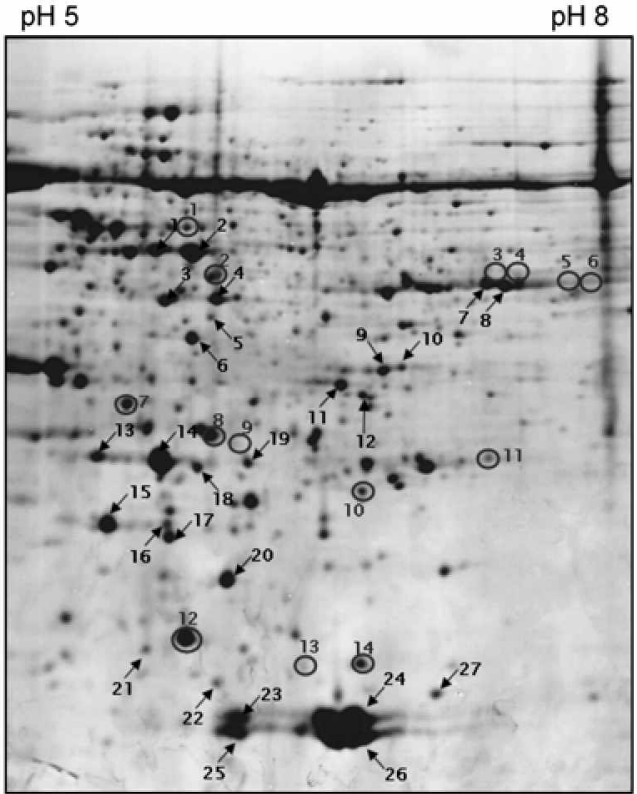

Figure 1. 2-DE map of control (a) and vemalization-treated t.t. thaliana (b). 
Table 1. Identified proteins with no major differences in both samples

\begin{tabular}{|c|c|c|c|}
\hline Spot No. & $\mathrm{MW} / \mathrm{pI}$ & Accession No. & Name \\
\hline 1 & $49069 / 7.55$ & gi|30687995 & RCA(Rubisco antivase) \\
\hline 2 & " & " & $"$ \\
\hline 3 & $41781 / 5.94$ & gi $\mid 79322651$ & fructrose-biphosphate aldolase \\
\hline 4 & $41318 / 9.07$ & gi| 42573227 & " \\
\hline 5 & $41630 / 8.13$ & gi| 15224351 & OASB: cysteine synthase \\
\hline 6 & $32096 / 5.46$ & $\operatorname{gi} \mid 21594017$ & putative lectin \\
\hline 7 & $36966 / 6.62$ & $\mathrm{gi} \mid 21593240$ & glyceraldehyde-3-phosphate dehydrogenase C subunit \\
\hline 8 & $36890 / 6.67$ & gil 15222166 & NAD binding/glyceraldehydes-3-phosphate dehydrogenase \\
\hline 9 & $47464 / 6.12$ & gi 27752811 & ribulose-1,5-bisphosphate carbosy lase \\
\hline 10 & $"$ & " & n \\
\hline 11 & $"$ & $"$ & $"$ \\
\hline 12 & $26054 / 6.04$ & gi|l 143394 & V-type proton-ATPase \\
\hline 13 & $28078 / 6.90$ & gil|15222166 & PSBP(oxygen-evolving enhancer protein 2): calcium ion binding \\
\hline 14 & $"$ & $"$ & " \\
\hline 15 & $47464 / 6.12$ & gi|27752811 & ribulose-1,5-bisphosphate carboxylase \\
\hline 16 & $25979 / 6.21$ & gi| 15233115 & LHCAl; chlorophyll binding \\
\hline 17 & $25653 / 9.04$ & gi||15233985 & hydrogen-transporting ATP sỵthase \\
\hline 18 & $23626 / 5.56$ & gi||15223576 & glutathione dehydrogenase (ascorbate) \\
\hline 19 & $25634 / 5.80$ & gi|| 18411929 & glutathione transferase 8 \\
\hline 20 & $28190 / 8.83$ & gi|15228674 & ROC4: peptidyl-prolyl cis-trans isomerase \\
\hline 21 & $16569 / 5.58$ & gi||15235002 & ATGRP8(glycine-rich protein 8); RNA binding \\
\hline 22 & $14490 / 584$ & gi|5881700 & ATPase epsilon suburit \\
\hline 23 & $20271 / 8.82$ & gi $\mid 15240901$ & ribulose-biphosphate carboxylase \\
\hline 24 & $"$ & $"$ & $"$ \\
\hline 25 & $14689 / 5.69$ & gil|13926229 & F1019.10/F1019.10 \\
\hline 26 & $"$ & $"$ & " \\
\hline 27 & $16229 / 7.03$ & gi|16396 & nucleoside diphosphate kinase \\
\hline
\end{tabular}

Table 2. Proteins differentially expressed in control and ventalization-treated AT

\begin{tabular}{|c|c|c|c|c|c|}
\hline \multirow{2}{*}{ Spot No. } & \multirow{2}{*}{$\mathrm{MW} / \mathrm{pI}$} & \multirow{2}{*}{ Accession No. } & \multirow{2}{*}{ Name } & \multicolumn{2}{|c|}{$\%$ Volume (std dev) } \\
\hline & & & & Control & Treated \\
\hline \multicolumn{6}{|c|}{ showing decrease } \\
\hline 11 & $24131 / 6.17$ & gi| 15224581 & ATGSTF9; glutathione transferase & $0.23(0.06)$ & $0.10(0.02)$ \\
\hline 1 & $51598 / 5.84$ & gi|l 15237059 & ATP binding/GTP binding/translation elongation factor & $0.21(0.02)$ & $0.09(0.01)$ \\
\hline 10 & $33871 / 8.73$ & gi| 18410804 & transcription factor & $0.28(0.07)$ & $0.09(0.02)$ \\
\hline 14 & $17507 / 5.91$ & gil 7269215 & putative major latex protein & $0.77(0.10)$ & $0.22(0.03)$ \\
\hline 5 & $37652 / 7.00$ & $\mathrm{gi} \mid 166702$ & glyceraldehyde-3-phosphate dehydrogenase A subunit & $0.23(0.17)$ & $0.04(0.03)$ \\
\hline 6 & $n$ & $"$ & " & $0.29(0.20)$ & $0.03(0.02)$ \\
\hline 13 & $17507 / 5.91$ & gi| 15236566 & unknown protein & $0.16(0.03)$ & n.d. \\
\hline 3 & $42820 / 8.16$ & gil 15222111 & NAD binding/glyceraldehyde 3-phosphate dehydrogenase & $0.09(0.06)$ & n1.d. \\
\hline 4 & $"$ & $"$ & $"$ & $0.16(0.12)$ & n1.d. \\
\hline 9 & $28166 / 5.29$ & gi 162320917 & carbonic anhydrase, chloroplast precursor & $0.10(0.04)$ & n.d. \\
\hline \multicolumn{6}{|c|}{ showing increase } \\
\hline 7 & $34858 / 8.37$ & gil 8404496 & unkn1own protein & $0.10(0.04)$ & $0.18(0.04)$ \\
\hline 2 & $38270 / 5.65$ & gi|l 5236768 & fructose-bisphosphate aldolase & $0.08(0.01)$ & $0.18(0.05)$ \\
\hline 8 & $27138 / 5.24$ & gil 742408 & triose phosphate isomerase & $0.18(0.03)$ & $0.41(0.15)$ \\
\hline 12 & $16880 / 5.85$ & gi| 15226605 & AtGRP7: RNA binding & $006(0.02)$ & $0.73(0.19)$ \\
\hline
\end{tabular}

teins (fructose-bisphosphate aldolase, triose phosphate isomerase, and an unhliown protein) showed about 2-fold increase. Most remarkable was AtGRP7: RNA binding protein. which showed more than 10 -fold increase in a repro- ducible manner as shown in Figure 3. The MALDI-TOF mass spectrum of AtGRP7 tryptic peptides is shown in Figure 4

To address if the increase of AtGRP7 protein is due to the 


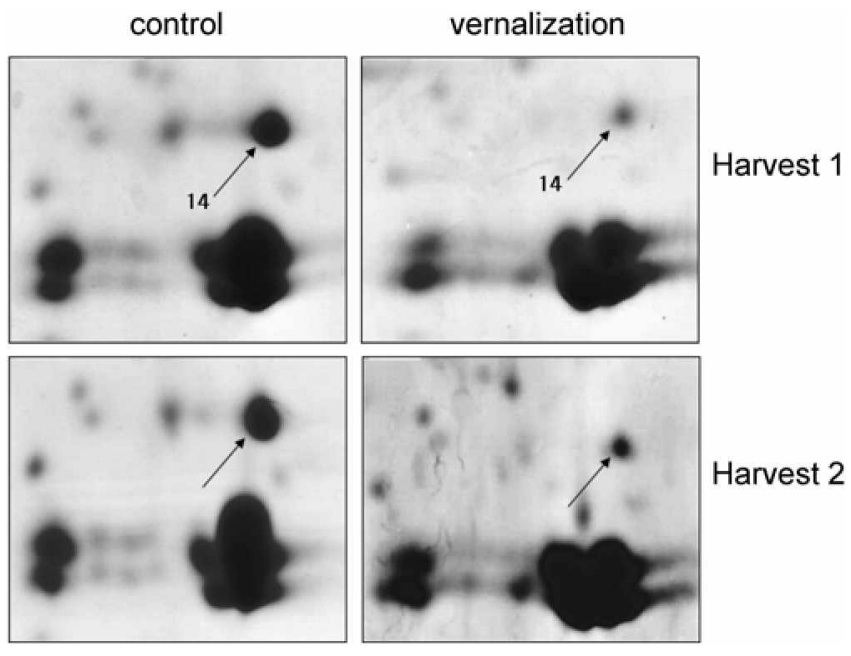

Figure 2. Reproducibility in the decreased expression of putative major latex protein.

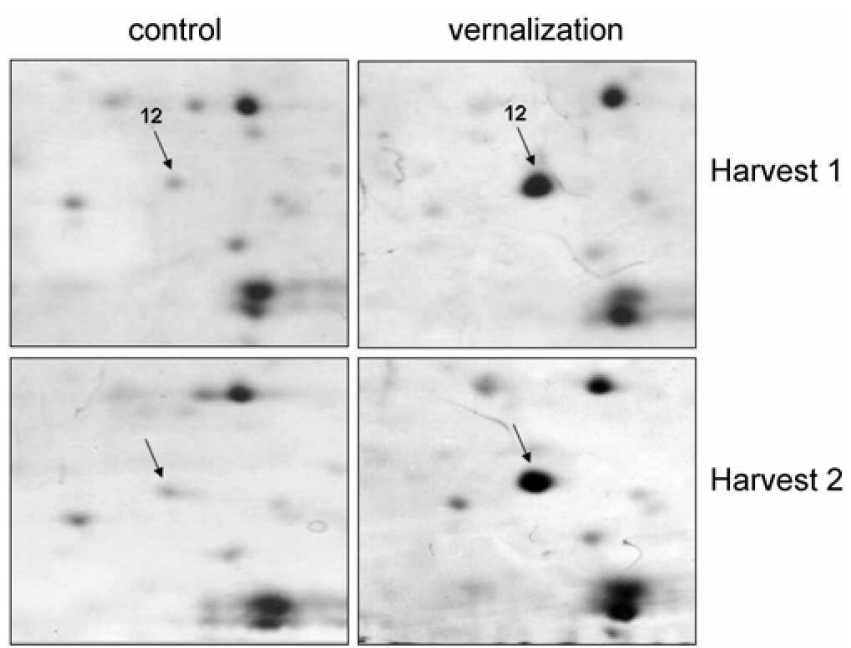

Figure 3. Reproducibility in the increased expression of AtGRP7; RNA binding protein.

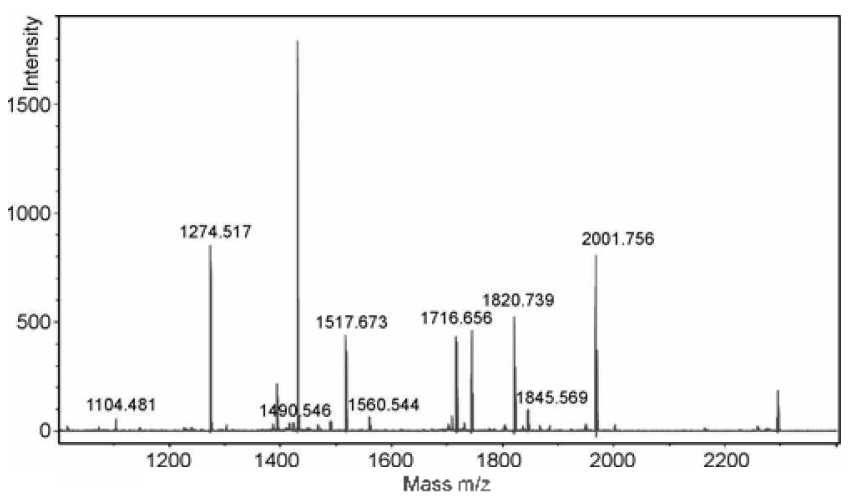

Figure 4. Mass spectrun of AtGRP7: RNA-binding protein.

increased transcript level, we performed reverse transcription-polymerase chain reaction (RT-PCR). As shown in Figure 5, AtGRP7 transcript was increased after l week cold treatment which does not affect the flowering time. In addition, such an increase was maintained for + weeks of vemalization treatment which strongly accelerates flowering

\section{$\begin{array}{llllll}0 & 1 & 2 & 3 & 4 & \text { weeks }\end{array}$}

AtGRP7

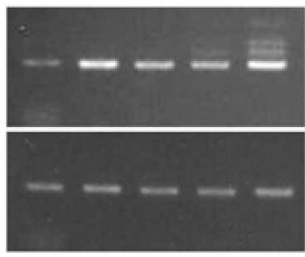

Figure 5. Expression level of AtGRP7 duning venalization treatment characterized using RI-PCR method.

time. It has been reported that $A t G R P 7$ transcript is increased by cold stress. ${ }^{7}$ The AtGRP7 gene has also been known to be involved in the circadian rlythm. ${ }^{8}$ thus suggesting the possible link between circadian rhythm and cold response. However. it has not been elucidated in the past if AtGRP7 is involved in the vernalization process or flowering time regulation

In contrast to AtGRP7. another member of the same gene family. AtGRP8. showed no significant change at the protein level upon vemalization, which was confimed by more than 3 independent biological replicates (Table 1). It is an interesting result because $A G G R P 8$ also has been reported to be induced by cold stress. ${ }^{7}$ When the plants were continuously exposed to cold the transcript level of AtGRP\& was increased during the first day but was gradually decreased afterwards. Thus, it is likely that AtGRP8 level is increased by short-term cold stress but gradually decreased during acclimation to a long period of cold. i.e.. vernalization. In contrast. AtGRP7 is not only induced by cold stress but also maintained during vernalization. Therefore it is likely that AtGRP7 has a divergent function compared to AtGRP8.

A variety of RNA binding proteins involved in posttranscriptional control are known. ${ }^{8-11}$ The RNA recognition motif (RRM) toward the N-teminal side of the RNA binding proteins consists of $80-90$ amino acids. The RNPl (RGFGFVTF) and RNP2 (CFVGGL) sequences in the RRM are directly involved in target RNA recognition and binding. Typical cellular RNA binding proteins have one or two RRM toward the N-teminal and various auxiliary motifs toward the C-terminal end. Auxilian motifs known include glycine-rich region, zinc finger motif. and acidic domain. ${ }^{11, i 2}$ The AtGRP7 protein that showed over 10-fold increase upon vemalization-treatment has a RNPI (RGFGFVTF) sequence at residue $49-56$ and a glycine-rich region toward the C-terminus as shown below.

1 MASGDVEYRC FVGGLAWATD DRALETAFAQ YGDVIDSKII NDRETGRSRG

51 FGPYTFKDEK AMKDAIEGMN GQDLDGRSIT VNEAQSRGSG GGGGHRGGG

101 GGYRSGGGGG YSGGGGSYGG GGGRREGGGG YSGGGGGYSS RGGGGGSYGG

151 GRREGGGGYG GGEGGGYGGS GGGGGW

Although vemalization is the same as cold treatment except for the length of the low temperature treatment. it is 
physiologically different in that the cold treatment usually does not affect flowering time significantly whereas vernalization affects flowering time dramatically. ${ }^{3}$ The molecular basis of vernalization has been elucidated recently. The final outcome of vernalization in Arabidopsis is the suppression of FLC, a strong repressor of flowering. Interestingly. many RNA binding proteins function as repressors of $F L C^{13}$ For example. $F C A$ gene encodes the protein with the RRM motif. ${ }^{14} F Y$ gene encodes polyadenylation factor, ${ }^{15}$ and $F P A$ and $F L K$ encodes proteins with plant-specific $\mathrm{KH}$ type RNA binding domain. ${ }^{10.17}$ The mutation of any of these genes causes a strong activation of $F L C$, thus resulting in late flowering. ${ }^{13}$ Our results showing increase in AtGRP7, a RNA binding protein. upon vernalization which causes the suppression of $F L C$ are consistent with the earlier results that many of the RNA binding proteins are involved in the repression of $F L C$. It has also been known that the cellular level of transcripts for RNA binding proteins is significantly increased under stress such as cold. drought. or viral infection. ${ }^{1 j, 11}$ Because AtGRP7 gene is also increased by cold stress. it is possible that AtGRP7 is involved in the response to cold stress. This question can be addressed by generating a loss-of-fiunction mutant and over-expression of transgenic line of AtGRP7.

Acknowledgment. We thank Hyung Kyoo Kwon for image analysis. Financial support through the BK21 program is appreciated.

\section{References}

1. Simpson1. G. G.: Dean. C. Science 2002. 296. 285-289.

2. Hayama. R.: Coupland. G. Plont Phusiolog! 2004. 135. 677684.

3. Chouard. P. Amual Review of Plon Phnsiologn 1960. H. 191238

4. Lang. A. Encyclopedia of Plan Physiolog, 1965, 15, 1371-1536.

5. Sung. S.: Amasino. R. M. Curent Opinion in Plant Biologv 2004. 7. $4-10$.

6. Lee. I.: Amasino. R. M. Plant Phwsiology 1995. 108. 157-162.

7. Kwak. K. T.: Kim. Y. O.: Kang. H. Jom al of Experimental Botan 2005. $56,3007-3016$.

8. Heintzn, D.: Nater, M.: Apel, K.: Staiger, D. Proceeding of National Acadeny of Science 1997, 94. $8515-8520$.

9. Burd. C. G.: Dreyfuss. G. Science 1994. 265.613-621.

10. Alba. M. M.: Pages. M. Trends in Plant Science 1998. 3. 15-21.

11. Sachetto-Martits. G.: Franco. L. O.: de Oliveria. D. E. Biochentica et Biophusica tcta 2000, 1492. 1-14.

12. Graumann. P. L.: Marahiel. M. A. Trends Biochem. Sci. 1998. 23. 286-290.

13. Quesada. V: Dean1. C.: Simpson. G. G. Int. J. Dev Biol. 2005. 19. 773-780.

14. Mackinight. R.: Bancroft. I.: Page. T.: Lister. C.: Schmidt. R.: Love. K.: Westphal. L.: Murphy, G.; Sherson. S.: Cobbett. C.: Dean. C. Cell 1997, 89,737-745.

15. Simpsont. G. G.: Dijkwel. P. P.: Quesada. V.: Hendersont. I.: Dean. C. Cell 2003. 13. 777-787.

16. Schomburg. F. M.: Patton. D. A.: Meinke. D. W. Amasino. R. A. Plam Cell 2001. 13. 1427-1436.

17. Lim. M.-H.: Kimn. I.: Kiml. Y.-S.: Chung. K.-S.: Seo. Y.-H.: Lee. I.: Kim. J.; Hong. C. B.: Kim. H.-J.; Park, C.-M. The Plant Cell 2004. $16,731-740$. 